\title{
Voces y Culturas Estudiantiles en la Escuela: Una Reflexión Teórico-Metodológica desde Procesos de Autoexploración de Barreras a la Inclusión Educativa
}

\author{
Student Voices and Cultures at School: A Theoretical- \\ Methodological Reflection from Self-Exploration Processes of \\ Barriers to Educational Inclusion
}

\author{
Ignacio Figueroa-Céspedes * \\ Cristopher Yáñez-Urbina \\ Universidad Diego Portales, Chile
}

\begin{abstract}
El presente artículo propone una reflexión a partir de una serie de experiencias de investigación-acción llevadas a cabo con distintos colectivos estudiantiles de escuelas municipales de la ciudad de Santiago de Chile. La finalidad consiste en sistematizar el desarrollo de procesos de autoexploración institucional a partir de la voz del estudiantado con una particular sensibilidad a las culturas en la escuela, entre ellas las culturas estudiantiles. En primer lugar, se analizan teóricamente los fundamentos y reflexiones producto de las experiencias desarrolladas, para luego profundizar en las implicancias para su consideración en los procesos de desarrollo escolar inclusivo. Como conclusiones se plantean las conexiones recíprocas que el enfoque en cuestión mantiene con el desarrollo cognitivo y la educación intercultural, planteándose como vector a partir del cual pensar la escuela en sus diversas dimensiones.
\end{abstract}

Descriptores: Educación integradora; Educación intercultural; Investigación participativa; Participación estudiantil.

This article proposes a reflection based on a series of research-action experiences carried out with different student groups of municipal schools in the city of Santiago de Chile. The purpose is to systematize the development of institutional selfexploration processes based on the student's voice with a particular sensitivity to cultures in school, including student cultures. In the first place, the foundations and reflections from the experiences are analysed theoretically, then deepen the implications for consideration in the processes of inclusive school development. As conclusions, the reciprocal connections that the approach in question maintains with the cognitive development and the intercultural education, arise as a vector from which to think the school in its diverse dimensions.

Keywords: Inclusive education; Intercultural education; Participatory research; Students participation. 


\section{Introducción}

$$
\begin{array}{r}
\text { Verdad, verdad mi verdad } \\
\text { no quiero tu autoridad } \\
\text { solo quiero caminar con dignidad } \\
\text { y conquistar mi libertad. }
\end{array}
$$

(Ana Tijoux, Canción, Mi verdad, 2012)

Durante el último tiempo la educación se ha visto involucrada con el desafío de dar respuestas a las problemáticas vinculadas con el reconocimiento y validación de la diversidad en los espacios educativos. En otras palabras, hablamos del desafío que plantea la inclusión social en educación como aquel proceso por el cual se busca identificar y superar aquellas barreras al aprendizaje y la participación, con la finalidad de responder a la diversidad (Echeita y Ainscow, 2011).

La transformación de los sistemas escolares en miras a un desarrollo escolar inclusivo sienta sus bases en la reflexión y trabajo sobre Políticas, Prácticas y Culturas. Siguiendo a Booth y Ainscow (2012), estos cambios deben inscribirse en el proyecto de la creación de culturas escolares inclusivas desafío no menor entendiendo que las culturas escolares son un complejo patrón de normas, actitudes, creencias, comportamientos, valores, tradiciones y mitos que se encuentran profundamente arraigados en el corazón de la organización escolar (Barth, 2002).

De tal forma, es necesario conducir un proceso de reculturación (Fullan, 2002) que cuestione y transforme la tradicional cultura escolar considerando la construcción y reflexión desde un marco ético-valórico inclusivo (Booth, 2003), superando problemáticas tales como la exclusión de la diferencia y las culturas de procedencia del estudiantado y el resto de la comunidad (Jorquera, 2015), un marcado adultocentrismo (Duarte, 2012), la proliferación de ambientes violentos (Abramovay, 2005), entre muchas otras.

Por lo tanto, cualquier iniciativa de desarrollo escolar que se denomine inclusiva debe considerar una aproximación metodológica que incorporen la multiplicidad de voces de sus diversos participantes, en el sentido de reconocer y validar las perspectivas de un amplio abanico cultural (Long, 2003), para la construcción de conocimiento que permita identificar barreras al aprendizaje y la participación. En otras palabras, es necesario ampliar la mirada más allá del monoculturalismo (Booth y Ainscow, 2012; Dietz, 2012) que se ha instalado en la escuela para encontrarse con las diversidades que cohabitan el espacio educativo como un lugar propiamente intercultural (Ferrão, 2010).

Este proceso de autoexploración y transformación requiere del reconocimiento de todas las personas que conforman la comunidad educativa, refundando la noción de colaboración, poniendo de manifiesto la necesidad de un posicionamiento para el cambio de valores. Esto implica la articulación y construcción sostenida de un tejido social sólido y una ética comunitaria basada en criterios de justicia, cuidado, crítica y responsabilidad (Escudero y Martínez, 2011).

Estas visiones han impulsado una serie de indagaciones sobre los procesos de participación en la gestión cotidiana de la escuela desde la necesidad de dar relevancia a la voz del estudiantado (Argos et al., 2011 ; Calvo, Haya y Susinos, 2012; Escobedo, Sales y Traver, 2017; Rojas, Haya y Lázaro-Visa, 2011). Dichas iniciativas coinciden con una serie de estudios previos llevados a cabo por nuestro equipo investigativo, en los cuales se concluyó que los estudiantes corresponden al colectivo que menos participa en los 
procesos de desarrollo escolar inclusivo (Figueroa y Muñoz, 2015; Figueroa, Soto y Sciolla, 2016; Soto, Figueroa y Yáñez-Urbina, 2017), además de existir un marcado privilegio por técnicas de producción de información de carácter estático y extensivo, tal como los cuestionarios y encuestas, lo que dificulta una participación deliberativa de los distintos actores en el proceso de cambio (Yáñez-Urbina, Figueroa, Soto y Sciolla, 2018).

Por lo tanto, en el presente artículo realizamos una reflexión teórico metodológica a partir de la experiencia del equipo investigador desarrollando distintas aproximaciones que rescatan las voces estudiantiles en iniciativas de cambio inclusivo, esto en colaboración con diversas instituciones de dependencia municipal en la ciudad de Santiago de Chile. Lo último, considerando las implicancias asociadas tanto a la forma de producir un conocimiento sobre la escuela, como a la necesidad de reconfigurar el campo cultural de la misma (Yáñez-Urbina, Figueroa, Soto y Sciolla, 2018). Tomamos como elementos de referencia las notas de campo realizadas por el equipo de investigación, entrevistas a estudiantes y el material producido por éstos, sistematizando cuatro fases metodológicas comunes a dichas experiencias. Es conveniente hacer notar que la investigación en la que se enmarca este artículo cuenta con la aprobación del Comité de Ética de nuestra Universidad, empleando los consentimientos informados y protocolos necesarios para la participación del estudiantado en este proyecto.

\section{Abordaje inicial: Notas sobre la voz del estudiantado}

Desde el plano histórico es imposible negar los vínculos existentes entre la educación, la difusión de los valores asociados al proyecto ilustrado y la conformación de los EstadoNación (Anderson, 2006). De ello se desprende que el conjunto de sus participantes se ha transformado en "sujetos subalternos, cuya voz y subjetividad es reemplazada por la voz que las elites gobernantes necesitan escuchar para el levantamiento del proyecto nacional" (Jorquera, 2015, p. 42).

En esta línea, el colectivo conformado por el estudiantado es de particular interés (Messiou, 2013), pues dentro de estas dinámicas son silenciados por una cultura profesionalista de parte de docentes y directivos (Escobar et al., 2017) que los invalida como sujetos autónomos y de derecho (Gascón y Godoy, 2015). De tal forma, el estudiantado se ha constituido como un grupo que históricamente ha sido subyugado por una lógica adultocéntrica que excluye del espacio público a todo aquello que no es inherentemente adulto. Por lo tanto, la voz del estudiantado no tiene cabida dentro de la institución escolar, siendo acallada y restringida al campo de la obediencia y la sumisión a normas y reglas (Duarte, 2012).

Frente a esta problemática, se instalan los debates en torno a la inclusión en la educación, la cual no se limita a la congregación de una diversidad estudiantil en el aula, sino que implica la necesidad de reflexionar en qué medida la diversidad (étnica, etaria, cultural, socioeconómica, género, etc.) transforma los presupuestos dominantes acerca del aprendizaje, las habilidades y el fin último de la educación (Booth y Ainscow, 2012; Echeita y Ainscow, 2011; Molina y Christou, 2009). Uno de los ejes particulares de este enfoque corresponde a la voz del estudiantado, como una noción que aglutina un conjunto heterogéneo de aportes que dotan de relevancia al rol político que desempeñan los y las estudiantes en las actividades educativas y organizacionales de sus propios contextos (Nieto y Portela, 2008; Susinos, 2012). 
Sin embargo, dentro de estas definiciones es posible encontrar una multiplicidad de enfoques y variaciones cuyas particularidades se centran más en la forma en la cuales las acciones son llevadas a cabo en sus diversas dimensiones, que en la elección de un tipo particular de abordaje por sobre otro (Susinos y Ceballos, 2012). Por lo cual, resulta necesario revisar brevemente algunas de las concepciones que son puestas en juego a la hora de hablar y de trabajar desde este enfoque.

El primer elemento a destacar consiste en aquel que responde a la pregunta ¿Quién puede producir una narrativa con respecto a la institución escolar? o, lo que es homologable a decir ¿Quién puede producir conocimiento sobre los contextos educativos, sus problemáticas, posibilidades y tensiones? Este elemento se asocia directamente en un dilema relacional, es decir, se instala en la disputa por el sentido y significado del mundo (Stange, 2007) en la intersección existente entre el campo de la comunicación y de la política (Gascón y Godoy, 2015; Salinas y Stange, 2006, 2009).

En estas coordenadas, la reflexión de la voz del estudiantado es tanto tributaria de las epistemologías feministas, las cuales se han preguntado por los cuerpos que importan (Butler, 2006), y la producción de conocimiento científico (Haraway, 1995); así como también del enfoque decolonial asociado a la voz de la subalternidad (Spivak, 2011) y la justicia cognitiva (Santos, 2018). De tal manera, se instaura una noción de crítica en contra de un sujeto cognoscente universalista que se identifique con la figura que desde estos linderos se ha reconocido como el "hombre blanco europeo". Noción sobre la cual es necesario incluir la etiqueta de "adulto" a partir de cómo es comprendida por el proyecto ilustrado que permea a la educación (Anderson, 2006; Jorquera, 2015).

En el breve ensayo Respuesta a la pregunta ¿Qué es la ilustración?, Immanuel Kant (1994) establece las coordenadas desde donde puede ser comprendido el proyecto filosófico que, tal como lo indica Ruidrejo (2006), involucra una moral de la libertad asociada exclusivamente al uso de la razón. En estas coordenadas, el filósofo alemán refiere al no uso de la razón como "minoría de edad", haciendo alusión a la infancia como antagonista del adulto, dotando a la primera de características peyorativas: no completa, incapaz del uso de la razón, necesidad de conducción, etc.

A partir de lo anterior, se instala un ethos que caracteriza a la institución escolar desde su incipiente implementación hasta la actualidad, la cual es atravesada por un constante proceso de aculturación de sus estudiantes para que logren insertarse dentro de los saberes hegemónicos y tengan acceso a aquel ideal del razonamiento propio de la ilustración (Jorquera, 2015). Siendo así, la infancia constituye una construcción histórica de reciente data a partir de la cual se desprende la noción de alumno como aquel sujeto que hay que formar para el futuro de la sociedad (Baquero y Narodowski, 1994).

En esta fórmula, del estudiantado como un adulto incompleto, lo considera como esencialmente pasivo frente a una cultura que lo permea, siendo un mero receptor y nunca un agente de cambio. Frente a esta concepción adultocéntrica (Pérez y Rincón, 2010), los nuevos estudios sociales de la infancia se proponen elaborar un plan de trabajo crítico, entendiéndola como un agente social y políticamente activo tanto en la reproducción como en la transformación social (Vergara et al., 2015).

De esta forma resulta necesario replantear las concepciones que se encuentran a la base de las prácticas tanto pedagógicas como de investigación en las escuelas desde el campo de los diseños metodológicos: el investigador debe participar como un aprendiz del mundo 
infantil (Vergara et al., 2015). Este último, en palabras de Jara (2018), configura un mundo poético y creativo en el cual "el des-orden establecido en el juego [y el dibujo] es un orden nuevo, un sistema semiótico, un lenguaje creado por niñas y niños para generar un relato" (p. 48).

Por lo tanto, los fundamentos desde donde se instala la noción de voz del estudiantado tienen lugar en un cruce que ya no solamente se pregunta por quién puede producir conocimiento sobre los contextos educativos, sino que también aborda la pregunta por el cómo producirlos desde las diversas voces que se encuentran cohabitando el espacio educativo y la multiplicidad de formas a partir de las cuales se puede narrar la escuela más allá de un ethos que pretenda la homogeneidad frente a una forma cultural hegemónica.

Dicho de otra forma, nuestro primer abordaje nos lleva inexorablemente a la necesidad de pensar a la escuela como un espacio en el cual es posible encontrar visiones del mundo tan diversas como las producciones que derivan de ellas. Hablamos de posicionar en el centro de nuestro análisis las pugnas, diálogos, luchas, alianzas y conflictos que existen entre el abanico de significaciones de la vida o culturas (García Canclini, 2007) que conviven en el espacio educativo. Un abordaje de estas características permite generar puntos de encuentro en la cual cada una de las voces es escuchada y valorada en su particularidad, produciendo espacios para la co-construcción de significados y el reconocimiento de estos mundos de vida estudiantiles (Ibáñez y Druker, 2018).

\section{Nuevos desafíos: Indagar colaborativamente desde la perspectiva de los actores}

Por lo pronto, nos vimos ante la necesidad de recalibrar los marcos desde los cuales mirar la escuela y sus dinámicas internas. Siguiendo los trabajos de Martín-Barbero (2002, 2011), encontramos en la intersección entre comunicación, política y cultura un punto fértil para el desarrollo de propuestas de cambio. De modo que, el presente apartado explora aquel sentimiento de pertenencia a una sociedad, el cual hace que los seres humanos se aproximen a una construcción colectiva de sentido, saberes, creencias y valores que sostienen un sentimiento de inclusión social (Calarco, 2006) sobre aquello que llamamos realidad (Calderón, 2015). Dicho de otra forma, a continuación, esbozamos los linderos para la puesta en marcha de una mirada inclusiva e intercultural de la diversidad escolar.

Antes de comenzar, es importante destacar que el abordaje que exponemos a continuación se distancia de aquellas perspectivas que analizan la cultura escolar privilegiando un abordaje managerial, homogeneizante y hegemónico hacia las producciones simbólicas y materiales. En su lugar, hemos construido una perspectiva heredada de los estudios culturales, los cuales pueden ser entendidos como un amplio paraguas conceptual que abarca una serie de perspectivas que tienen como puntos en común un rechazo a la distinción entre alta y baja cultura, y la comprensión de su campo de estudio como las prácticas y sus interrelaciones con la vida cotidiana (Hall, 2006). De tal forma, enfoca su estudio en la articulación de las prácticas con y desde la cultura, además de la posibilidad de transformar las relaciones de poder que en ellas se ejercen (Grossberg, 2009; Rosas, 2012).

En esta línea, el trabajo con el estudiantado responde a la pregunta por "dónde y cómo la gente experimenta sus condiciones de vida, las define y responde a ellas” (Hall, 2006, p. 
242) y tiene que ver con dos elementos fuerza, a saber: (1) el análisis sobre las barreras al aprendizaje y la participación desde sus propias experiencias y perspectivas; y (2) la generación de las condiciones para la visibilización de lo que hemos denominado como culturas estudiantiles, compuestas por la heterogeneidad de prácticas socio-históricas de los miembros del colectivo que, a su vez, responden a las interacciones entre culturas infantiles y culturas juveniles frente a la disposición de normas, valores y ritos presentes en la escuela, docentes, padres y políticas públicas.

No obstante, nos vemos ante la dificultad de encontrar una definición clara y precisa de culturas estudiantiles, esto debido a una serie de razones. En primer lugar, en la bibliografía especializada, su uso tiende a ser periférico y, desde allí, su definición es ambigua o inexistente (Cervini, 2003; Levinson, 1999), incluso se emplea como un homólogo al concepto de cultura escolar (Werner, 2009). Por otro lado, se privilegia su empleo como categoría de análisis en los contextos de educación superior (Brunner, 1985; Cortés y Kandel, 2002; Guzmán, 2002; Ordóñez, Mejía y Castellano, 2006), dejando al descubierto un sesgo en la investigación en educación en la cual está presente la idea de que los estudiantes de educación básica y media no (re)producirían culturas.

Frente a esta tendencia, nuestra pretensión no es elaborar una definición estática y canónica de las culturas estudiantiles, todo lo contrario, privilegiamos su conceptualización dinámica, dúctil, flexible y situada a cada uno de los contextos e interacciones en las cuales se encuentra envuelta. Así, tomamos dos ejemplos para su conceptualización, a saber: (1) la referencia al mundo de la música, ya fuese a partir de las letras de canciones o del recurso de la figura de algunos cantantes; y (2) expresiones lúdico-artísticas como formas de sistematizar sus impresiones y experiencias, las cuales en ocasiones desbordan el plano de los lingüístico para situarse dentro y en conexión con el mundo de las imágenes y la corporalidad.

En primer lugar, la música se ha hecho presente en todas las experiencias de trabajo desarrolladas como un sustrato o referencia cultural a partir del cual el estudiantado toma elementos que son pertinentes de expresar y resaltar en los procesos de autoexploración institucional. Así es como han contextualizado el mismo espacio a partir de la canción "Mi verdad" de la cantante Ana Tijoux para hacer referencia a cómo es su propia experiencia la que es tomada en cuenta y las posibilidades que ésta plantea en un proceso de cambio que busca la emancipación del sujeto como eje de desarrollo.

Llegado este punto, es necesario destacar que la así llamada música popular históricamente ha operado tanto como forma de construcción identitaria a la vez que resistencia histórico-cultural de los grupos subalternos frente a la hegemonía (Albarces, 2008). Asimismo, como destaca Castiblanco (2005), los géneros urbanos no se limitan solamente al plano musical, sino que lo desbordan como una forma de ser joven en donde cohabitan códigos nuevos tanto de lenguaje como de vestimenta, usos de los espacios, significación de la interacción colectiva, entre otros. Por lo tanto, y siguiendo nuestra propuesta analítica, es imposible negar estos elementos en cuanto constituyen una ampliación de los repertorios de lectura de la escuela cuando son manifestados por parte del estudiantado.

En cuanto a la segunda arista trabajada, se vuelven patentes una serie de elementos, tales como el juego, el dibujo y la fotografía, como vías alternativas a la comunicación oral para el planteamiento de la experiencia en la escuela. Cada uno de estos elementos son abordados en el siguiente apartado. No obstante, cabe resaltar que, siguiendo a Jara (2018), 
corresponden a códigos, lenguajes y metáforas utilizadas por niños, niñas y jóvenes como un orden desde el cual poder expresar sus ideas, sentimientos y opiniones, considerando sus voces como un proceso continuo, situado y negociado en un espacio en disputa (Taylor y Robinson, 2009).

De suma importancia es, entonces, situar una mirada que esté atenta cómo se ha construido una diferencia generacional en torno a los códigos utilizados (Berardi, 2007), lo que podría reflejar un cambio en la sensibilidad a partir del uso (y no la aparición) que le es dado a las nuevas plataformas multimediales que llevan a que, muchas veces, las producciones culturales sean en sí un collage de textos, imágenes, referencias e imaginarios de diversas procedencias (Scolari, 2008).

Así, entendemos que las culturas estudiantiles se presentan en términos de una relación tanto de diálogo como debate con la escuela entendida como un gran entramado de múltiples culturas que cohabitan un espacio. Siendo necesario ampliar la mirada hacia la interacción de las culturas en la escuela, como una amplia diversidad que no se limita al colectivo estudiantil, sino que se extiende a todos los miembros de la comunidad desde el personal no docente y padres hasta el equipo directivo, pasando por los profesores (Stoll y Fink, 1999)

Por lo tanto, se vuelve necesario desarrollar una estrategia que no solamente haga visibles estas voces y culturas, sino que también proponga cómo generar espacios para producir un diálogo entre las culturas estudiantiles y el resto de culturas que habitan la escuela proporcionando información útil acerca de las barreras al aprendizaje y la participación observadas por los distintos colectivos. En otras palabras, necesitamos facilitar espacios intersticiales, interculturales, que posibiliten la construcción colaborativa de conocimientos a partir de la necesaria negociación entre las diversas formas de experimentar y habitar la escuela.

\section{Espacios intersticiales como autoexploración: Construyendo una metodología centrada en la voz y escucha de los actores}

El desafío por producir espacios de diálogo entre la multiplicidad de culturas que existen en la escuela, tal como plantea Baeza (2008), exige una capacidad de mayor escucha y, por, sobre todo, de preparación técnica para visibilizar las voces que se encuentran silenciadas en los espacios educativos. Además, implica reconocer el lenguaje propio que utilizan, para luego aprehenderlo y fomentar el diálogo y la discusión. Tal como plantea Long (2003), ello despliega un proceso de negociación de recursos y significados, generando un movimiento co-construido con los diversos actores, es decir, se piensa en la creación de una intervención planeada.

Por estas razones, en el presente apartado detallamos una propuesta metodológica derivada de nuestra reflexión inicial, la cual ha sido llevada a cabo por el equipo de investigación para la gestión de espacios de diálogo e intercambio con miras a la construcción colectiva de conocimientos sobre la escuela sensibles a la multiplicidad cultural. Nuestro interés no radica en determinar unos abordajes por sobre otros, sino que, todo lo contrario, abrir las posibilidades para abordar la inclusión educativa desde una perspectiva que sea acorde a los contextos situados. 
Con la finalidad de simplificar su comprensión, hemos dividido el análisis de las propuestas llevadas a cabo en cuatro grandes etapas, los cuales no necesariamente son secuenciales, pues en muchas ocasiones operan en forma paralela. Estos son:

\subsection{Etapa 1: Convocatoria y construcción del espacio}

Como punto de partida, el equipo de investigadores realiza un contacto inicial con las escuelas presentando los aspectos centrales de la propuesta desde un enfoque ligado al desarrollo inclusivo de los contextos educativos. Las instituciones que aceptan (o solicitan) participar muestran una abierta disposición a iniciar procesos de autoevaluación, aunque en la práctica se hace necesario lidiar con algunos aspectos ambivalentes ante la presencia de voces disidentes (por ejemplo, silenciamiento de la disidencia, ver Figueroa et al., 2016).

El inicio de todos los proyectos tiene como gran hito la realización de una convocatoria abierta a todas y todos los/as miembros/as del colectivo con el cual se trabaja, en nuestro caso destaca el estudiantado. Esta actividad se caracteriza por ser voluntaria, otorgando la mayor cantidad de información posible y acompañada de la elaboración de un material visual que acompañe al llamado (figura 1).

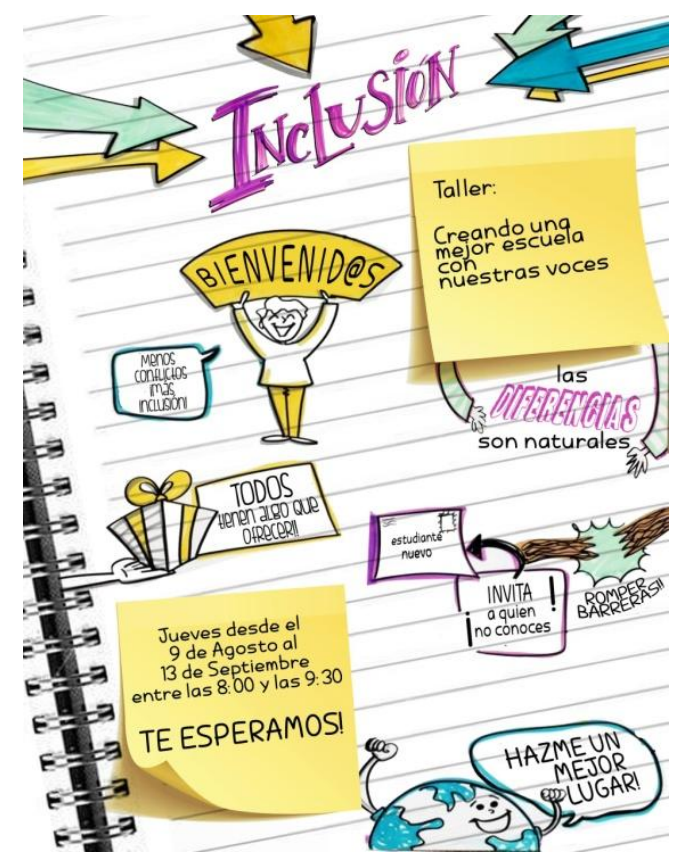

Figura 1: Flyer convocatoria

Fuente: Elaboración propia.

Una vez que se logra convocar a un número significativo de participantes se procede a coordinar un horario de trabajo que responda a la disponibilidad tanto del colectivo, como de la propia escuela en términos de espacios físicos y apoyo institucional. Con ello resuelto, las primeras sesiones tienen como finalidad la construcción de un grupo de trabajo como investigadores/as de la escuela (Sañudo y Susinos, 2018), se realizan dinámicas y actividades ligadas al conocimiento grupal, el empoderamiento y la necesidad de desarrollar lógicas de trabajo colaborativas para la producción de un conocimiento que sea dispuesto a la transformación de las dinámicas.

Finalmente, por medio de los diálogos, dinámicas y códigos comunicativos puestos en juego durante esta etapa inicial, se crean de forma colaborativa aquellos instrumentos de 
producción de información que serán utilizados más adelante. Éstos tienen en consideración los intereses, motivaciones, capacidades y referentes culturales de cada uno/a de los/as miembros/as del equipo de trabajo; además de caracterizarse por estar fuertemente vinculados con las artes visuales y escénicas, tal como sería el caso de la fotografía, el dibujo, las dramatizaciones, entre otras.

\subsection{Etapa 2: Indagación colaborativa: Investigando (en) la Escuela}

Posteriormente se procede al desarrollo mismo del proyecto como una instancia de diagnóstico participativo. En este desarrollo, es importante consignar que se trata de un proceso co-construido con el estudiantado, que requiere de la generación de condiciones mínimas para la producción de significados e investigaciones sobre sus contextos. Entre estas condiciones se encuentran la generación de un clima de seguridad, confianza y respeto entre los participantes.

Aquí son relevantes dos aspectos principalmente: por un lado, el diseño y ejecución de una propuesta de abordaje; y, por el otro, el análisis de la información producida. En primer lugar, los/as miembros del colectivo ejercen un rol de estudiantes-investigadores (Messiou, 2013), pues colaboran tanto en un plano de informantes como de indagadores de sus contextos, utilizando como inspiración la idea de estudiantes etnógrafos (Hohti, Karlsson y Palmu, 2013). En dicho sentido, su participación suele consistir tanto en otorgar su propia perspectiva de los procesos como la de consultar con sus pares y enriquecer la autoevaluación institucional.

Por otro lado, los análisis consisten en categorías que emergen desde el colectivo y que son plasmadas en producciones de diversos tipos y con referencias a sus propias formas de construir sus mundos de vida. A modo de ejemplo, podemos exponer la creación de un lienzo realizado en una escuela (figura 2) con la consigna "La escuela pequeña pero grande" que representa la limitación física de la escuela, pero su potencialidad en cuanto a proyecto educativo; y la producción de dos infografías (figura 3), a partir de la metodología de "Fotovoz", centradas en develar la necesidad de tranquilidad y diversión que perciben los/as estudiantes en un contex to de alta vulnerabilidad social (Yáñez-Urbina et al., 2018).

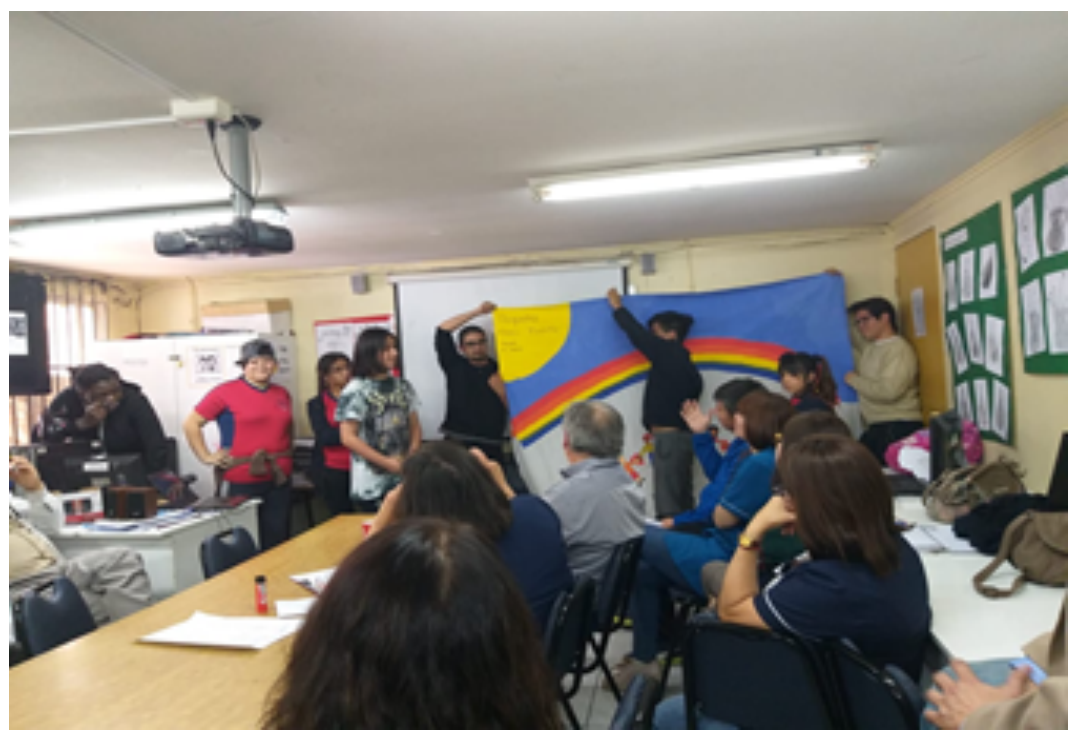

Figura 2: Lienzo "La escuela pequeña pero grande" Fuente: Elaboración propia. 
Esta serie de procesos tiende a producir una conciencia crítica por parte del estudiantado, considerando la necesidad de trabajar con todos los miembros de la comunidad educativa. En una ocasión, trabajando sobre la metáfora de la escuela como una máquina a través de

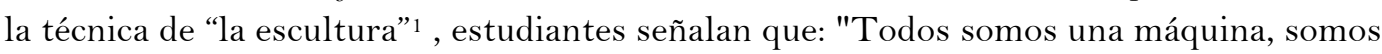
como un equipo, si falla una pieza falla la máquina". La caracterización de la escuela desde esta perspectiva, también conlleva la visibilización de cómo opera su propio colectivo, así un estudiante señala que: "Nos falta más comunicación entre nosotros, en el colegio hay muchos grupos separados, cuando tenemos talleres funcionamos como equipo, pero en el colegio no es tan así".
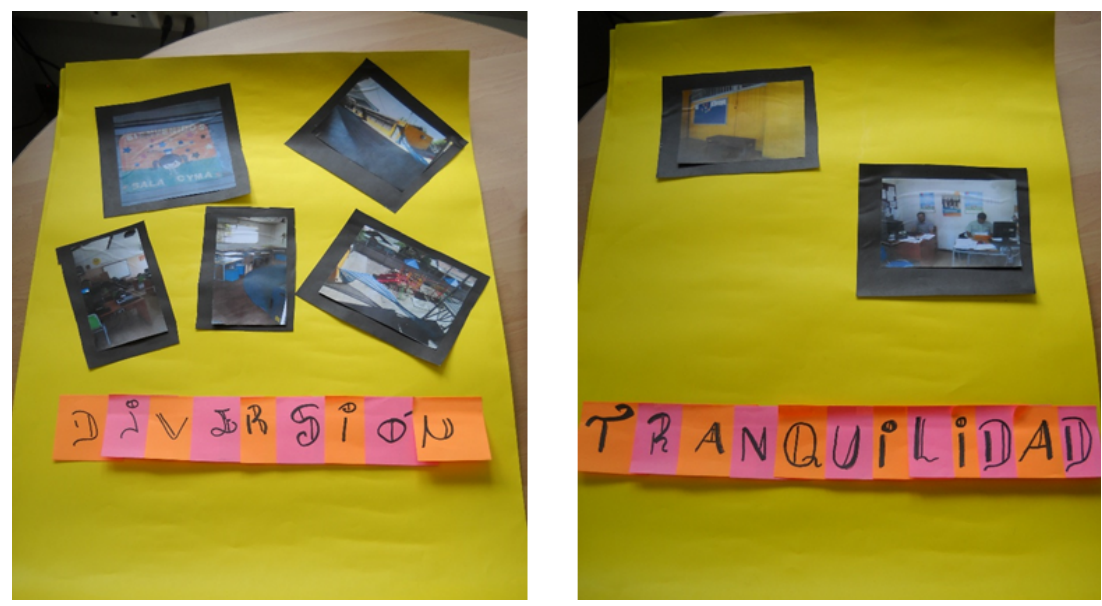

Figura 3: Infografías Tranquilidad y Diversión

Fuente: Elaboración propia.

\subsection{Etapa 3: El extranjero como amigo crítico}

De forma paralela y transversal a las etapas anteriores y la siguiente, el equipo externo desarrolla procesos autorreflexivos en relación a su rol en la escuela como un coinvestigador colaborativo y mediador con el equipo directivo y el resto de la comunidad educativa.

De tal forma, coopera con el equipo de trabajo por medio de una facilitación grupal, así como también realizando preguntas ingenuas para profundizar en qué es lo que se desea expresar y mostrar las tensiones que pueden estar operando entre las visiones de los/as estudiantes-investigadores. Estas intervenciones, dialógicas e inmersivas, posibilitan la emergencia de la reflexividad y en particular la autorreflexividad acerca de sus propios mundos de vida y de sus visiones sobre la participación en la escuela.

Por otro lado, el amigo crítico en conjunto con su contraparte de la escuela, colabora en la gestión de tiempos, espacios, materiales y reuniones que son necesarias para el óptimo desarrollo del proyecto. Esta consiste en una actividad eminentemente política al tratar de conciliar expectativas de la escuela en su conjunto y problematizar prácticas del colectivo adulto para preparar las condiciones mínimas para el fomento del diálogo trans-

${ }_{1}^{1}$ Técnica psicodramática que implica la escenificación de una imagen mental compartida, desarrollada a través del cuerpo humano u objetos, moldeando en este caso una percepción grupal (Rojas-Bermúdez, 1997; Reyes, 2007). 
colectivo, así como también en la conformación de prácticas de escucha y de "amistad crítica" que sean sustentables en el tiempo.

\subsection{Etapa 4: Diálogos participativos: explorando la "Zona de contacto"}

Finalmente, los proyectos desembocan en la co-construcción de un espacio en el cual el colectivo de estudiantes-investigadores pueda debatir y compartir sus ideas con profesores, funcionarios, padres y apoderados, y directivos (figura 4). Ello es fundamental para el desarrollo de una cultura abierta a la multiplicidad de voces que cohabitan el contexto educativo. Aquí, el estudiantado presenta los resultados de sus exploraciones y se discute en torno a ellos facilitando la negociación de significados y perspectivas sobre la escuela, evitando en todo momento las interpretaciones arbitrarias y convenientes a algún plan previamente orquestado por los adultos, incluyéndonos en nuestra condición de investigadores. De esta forma, el abordaje orienta hacia una indagación conjunta de las culturas estudiantiles, propiciando escenarios para la validación de dicho proceso de producción de conocimiento.

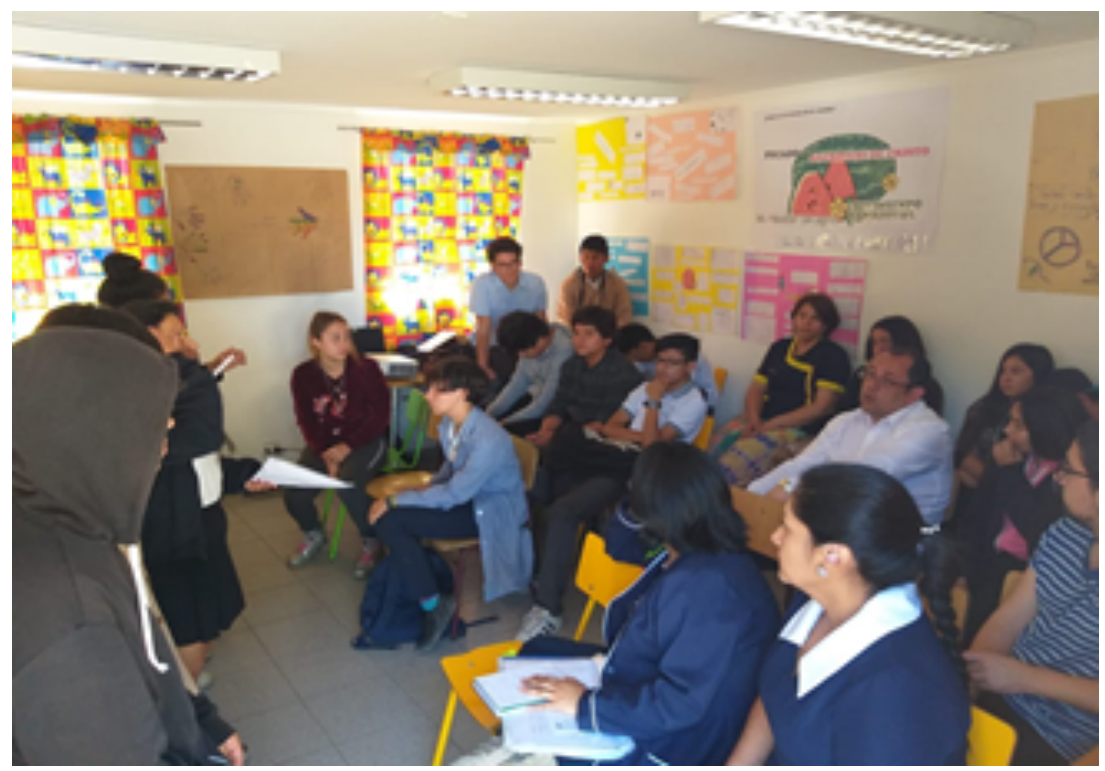

Figura 4: Diálogos participativos

Fuente: Elaboración propia.

En este espacio seguimos la sugerencia de MacPheil (2010) relacionada con la preparación de un contexto de apertura y confianza que posibilite que los adultos procesen conceptos más allá de las concepciones tradicionales acerca de la enseñanza y el aprendizaje en la escuela, con la expectativa de producir un diálogo constructivo. En este sentido, resulta relevante lograr en los adultos participantes una actitud de genuina escucha, para lo cual es relevante transitar desde una racionalidad técnica a una racionalidad crítica (Sañudo y Susinos, 2018), de forma tal de proporcionar reconocimiento a las perspectivas del estudiantado y construir un conocimiento situado y relevante. En este sentido, son los propios estudiantes quienes lideran la conversación, exponiendo sus narrativas y producciones culturales. El grupo de adultos escucha, acoge y procesa las barreras al aprendizaje y la participación que emergen desde sus perspectivas. 


\section{Conclusiones}

Hasta el momento hemos revisado las reflexiones tanto teóricas como prácticas a las cuales, como equipo de investigación, hemos llegado luego de una serie de experiencias trabajando con grupos de estudiantes en el ejercicio de relevar sus voces en contextos de desarrollo escolar inclusivo. Por lo tanto, nos queda concluir acerca de las implicancias de estas experiencias que buscan relevar las voces y culturas estudiantiles.

En primer lugar, cabe destacar la necesidad (y relevancia) de realizar un trabajo que dote de agencia política al estudiantado en el marco de una educación para la ciudadanía y la democracia. Las metodologías que hemos utilizado desde este enfoque pretenden combatir miradas que conceptualizan a los estudiantes como sujetos pasivos incapaces de reflexionar sobre su entorno y mucho menos transformarlo. En este sentido, el estudiantado no reproduce de forma acrítica una idea hegemónica de cultura, sino que también es capaz de construir y apropiarse participativamente (Rogoff, 1997) de los sentidos últimos de una cultura transformadora, intercultural e inclusiva.

Por otro lado, el estudiantado no solamente es un agente válido de conocimiento, sino que además éste no se reduce a una concepción racionalista de la escuela, sino que involucra una experiencia integral, emocional y hasta estética de sus propias vivencias. En otras palabras, desde esta óptica reconocemos la diversidad de formas de conocer y de expresar dicho conocimiento sobre la escuela, por sobre una homogeneización de las narraciones en un plano monocultural que responde a códigos objetivables en clave de efectividad.

Asimismo, las experiencias nos muestran que el estudiantado no sólo ejerce una crítica hacia las condiciones de su escolarización, sino que también reflexionan sobre sus propias prácticas y las del colectivo en general, logrando examinar elementos de sus culturas. Cuestionan su involucramiento en la toma de decisiones y la participación en los espacios que les han sido otorgados, las formas en las cuales se relacionan y, finalmente, su rol en los procesos de enseñanza-aprendizaje.

En el desarrollo de estas experiencias, el estudiantado logra elaborar procesos metacognitivos de alta complejidad al observar su entorno y sus propias prácticas culturales; flexibilizar sus patrones para adaptarse a una serie de situaciones y de problemáticas que se presentan a lo largo del desarrollo; escucha activa, reflexión y crítica de otras perspectivas; sintetizar y materializar sus acuerdos y opiniones en insumos dispuestos para la discusión; etc. Lo anterior sugiere que este trabajo de investigación acción colaborativa, no solamente tiene repercusiones en términos de la educación para la democracia y la inclusión social, sino que se instala como un vehículo para el desarrollo cognitivo sustantivo y transformador de sus entornos.

De modo que, resulta necesario también reflexionar respecto a las implicancias pedagógicas de este abordaje, las cuales abarcan tanto la mediación frente a una pluralidad de formas de experimentar el mundo, y los requerimientos de una formación docente sensible a las culturas en la escuela. Resulta relevante dar cuenta, entonces, de aquellos sistemas de conocimiento (Long, 2007) que se construyen y reconstruyen en la interacción social, propiciando su escucha desde la participación (Rogoff, 1997) y desde un profesionalismo docente de carácter dialógico que conecte y valide la pluralidad de voces que participan en la experiencia pedagógica (Figueroa, 2020). En este proceso, es necesario estar consciente del riesgo pedagógico (e investigativo) que proviene de la costumbre de buscar respuestas "expertas" a las problemáticas de la diversidad, sin 
considerar las perspectivas locales, operando como una "exclusión incluyente" (Gentili, 2011) que en este sentido construye de forma acrítica "sistemas de ignorancia" sobre la otredad (Long, 2007) esencializando las diferencias y despolitizando, en este caso, la posibilidad transformadora del estudiantado.

Es necesario profundizar en las implicancias que tiene entender la escuela como un hábitat megadiverso en la que las distintas culturas interactúan entre sí, ya sea por medio de intercambios, relaciones de poder y jerarquización, en disputas o alianzas las unas frente a las otras, involucrando a todos y cada uno de los colectivos presentes en ella (Taylor y Robinson, 2009). Por lo tanto, en la búsqueda de un enfoque consistente, los dispositivos que hemos explorado buscan aproximarse a la zona de contacto (Allan, 2007): espacio de confrontación/encuentro cultural, productivo y dinámico, sensible a los procesos de escucha, colaboración y "amistad crítica" que hemos descrito. Para ello pensamos el entramado cultural de la escuela como el proceso continuo del tejido de un telar: aquí urdimbres y tramas son las diversas culturas, cada una de las cuales genera un amplio abanico de interacciones que tienen como resultado un telar que puede ser resistente o débil, con o sin diseños, etc. Asimismo, arrastra una historicidad de relaciones (aquellas voces que reverberan) que la conformaron, las cuales pueden ser transformadas dando escucha y acción a las narrativas estudiantiles, permitiendo procesar y abordar los posibles malestares que emerjan en la experiencia.

Esta metáfora del tejido del telar, sin embargo, plantea una serie de interrogantes ${ }_{i} E s$ posible remirar los aportes del enfoque intercultural para abordar la multiplicidad de culturas y voces en la escuela? ¿Existe algún riesgo en la manera en la cual se ha considerado dicho enfoque? En la actualidad, los abordajes de la escuela desde un enfoque intercultural han centrado sus preocupaciones en dos focos: los fenómenos migratorios y la reivindicación de las etnias y/o pueblos originarios. Ambos problematizados al inscribirse en contextos educacionales homogeneizantes y sin una apertura cultural a la diversidad. No obstante, en su operacionalización se han centrado enfáticamente sobre grupos en específico, realzando su caracterización en tanto que diferentes y necesitados de ayuda y un trato especial.

Vemos, entonces, cómo el enfoque intercultural de corte funcional (Ferrão, 2010) corre el mismo riesgo que explican Echeita y Ainscow (2011) al respecto de una educación inclusiva centrada puramente en el abordaje a las necesidades educativas especiales. En ambas situaciones, lejos de combatir las desigualdades, éstas son producidas por medio de la discriminación positiva, el despliegue de una serie de programas, proyectos y profesionales focalizados en otorgar ayuda asistencialista o desde marcos prescriptivos que rigidizan el dinamismo presente en el intercambio cultural.

Frente a esta problemática paradojal del enfoque intercultural, es necesario recurrir a una perspectiva crítica la cual, siguiendo a Ferrão (2010), plantea el desafío de extender la mirada de la diferencia hacia el conjunto, acentuando su positividad y la pluralidad de formas de aprehender la realidad, sin recurrir a la estigmatización de un grupo en particular. Dando cuenta del carácter diverso y contextual de las identidades culturales que coexisten en la escuela, nuestro enfoque plantea una perspectiva dinámica que cuestiona la esencialización de las diferencias y contempla la dimensión conflictiva como natural a este tipo de interacción (Dietz, 2012). En dicho sentido, educación inclusiva y educación intercultural encuentran un punto de intersección y complementariedad, al 
reconocimiento y exploración dialógica del entramado de voces y culturas que cohabitan el espacio educativo.

En suma, presentamos un enfoque de autoexploración sensible a las culturas estudiantiles que, amplía el fundamento de cambio educativo en miras a una educación interculturalinclusiva (Sales-Ciges, 2017), cuestiona abiertamente la hegemonía adultocéntrica (Duarte, 2013), y avanza hacia la emergencia de una cultura descolonizada, renovada y novedosa bajo los principios de igualdad de derechos, reconocimiento y respeto mutuos (Liebel, 2016). En este sentido, es necesario crear propuestas que propicien el desarrollo cognitivo como un recurso transformador; al mismo tiempo que es necesario relevar la mirada ético-política presente en un enfoque intercultural crítico, en torno a las diferencias culturales extendidas y no centralizadas en un grupo en particular. Por lo tanto, es posible pensarlo como un vector a partir del cual desarrollar metodologías y enfoques que permitan construir culturas, políticas y prácticas inclusivas.

\section{Referencias}

Abramovay, M. (2005). Violencia en las escuelas: un gran desafío. Revista Iberoamericana de Educación, 15, 53-66.

Albarces, P. (2008). Posludio: Música popular, identidad, resistencia y tanto ruido (para tan poca furia). Trans. Revista Transcultural de Música, 12, 15-34.

Allan, J. (2007). Rethinking inclusive education: The philosophers of difference in practice. Dordrecht: Springer.

Anderson, B. (2006). Comunidades imaginadas. Reflexiones sobre el origen y la difusión del nacionalismo. Ciudad de México: Fondo de Cultura Económica.

Argos, J., Ezquerra, P. y Zubizarreta, A. (2011). Escuchando la voz de la infancia en los procesos de cambio e investigación educativos. Aproximación al estudio de las transiciones entre las etapas de educación infantil y educación primaria. Revista Iberoamericana de Educación, 54(6), 1-18. https://doi.org/10.35362/rie5451651

Ascorra, P., López, V. y Urbina, C. (2016). Participación estudiantil en escuelas chilenas con buena y mala convivencia escolar. Revista de Psicología, 25(2), 1-18.

Baeza, J. (2008). El diálogo cultural de la escuela y en la escuela. Estudios Pedagógicos, 34(2), 193206. https://doi.org/10.4067/S0718-07052008000200012

Baquero, R. y Narodowski, M. (1994). ¿ Existe la infancia? Revista del Instituto de Investigaciones en Ciencias de la Educación, 3(6), 61-67.

Barth, R. (2002). The culture builder. Educational Leadership, 59(8), 6-11.

Berardi, F. (2007). Generación post-alfa: Patologías e imaginarios en el semiocapitalismo. Buenos Aires: Tinta Limón.

Booth, T. y Ainscow, M. (2012). Guía para la inclusión educativa: Desarrollando el aprendizaje y la participación en las escuelas. Santiago de Chile: CSIE-FCF.

Brunner, J. (1985). El movimiento estudiantil ha muerto. Nacen los movimientos estudiantiles. Santiago de Chile: FLACSO

Butler, J. (2006). Deshacer el género. Barcelona: Paidós.

Calarco, J. (2006). La representación social de la infancia y el niño como construcción. Recuperado de http://www.bnm.me.gov.ar/giga1/documentos/ELOO1729.pdf. 
Calderón, D. (2015). Los niños como sujetos sociales: Notas sobre la antropología de la infancia. Nueva Antropología, 28(82), 125-140.

Calvo, A., Haya, I. y Susinos, T. (2012). El rol del orientador en la mejora escolar. Una investigación centrada en la voz del alumnado como elemento de cambio. Revista de Investigación en Educación, 10(2), 7-20.

Castiblanco, G. (2005). Rap y prácticas de resistencia: una forma de ser joven. Reflexiones preliminares a partir de la interacción con algunas agrupaciones bogotanas. Tabula Rasa, 3, 253-270. https://doi.org/10.25058/20112742.237

Cervini, R. (2003). Relaciones entre composición estudiantil, proceso escolar y logro en matemáticas en la educación secundaria Argentina. Revista Electrónica de Investigación Educativa, 5(1), art. 4.

Cortes, C. y Kandel, V. (2002). Reflexiones en torno a las nuevas formas de participación estudiantil en la vida política de la universidad. Fundamentos en Humanidades, 3(5), 23-34.

Dietz, G. (2012). Multiculturalismo, interculturalidad y diversidad en educación: Una aproximación antropológica. Ciudad de México: Fondo de cultura económica.

Jara, I. (2018). Adultocentrismo y género como formas negadoras de la cultura infantil. Revista Saberes Educativos, 1, 47-67.

Duarte, C. (2012). Sociedades adultocéntricas: Sobre sus orígenes y reproducción. Última Década, 2O(36), 99-125. https://doi.org/10.4067/S0718-22362012000100005

Echeita, G. y Ainscow, M. (2011). La educación inclusiva como derecho. Marco de referencia y pautas de acción para el desarrollo de una revolución pendiente. Tejuelo, 12, 26-46.

Escobedo, P., Sales, A. y Traver, J. (2017). La voz del alumnado: su silencio y la cultura profesionalista. Educación XXI, 2O(2), 299-318. https://doi.org/10.5944/educxx 1.19045

Farías, A. (2003). El difícil camino hacia la construcción del niño como sujeto de derechos. Revista de Derechos del Niño, 2, 187-224.

Ferrão, V. (2010). Educación intercultural en América Latina: Distintas concepciones y tensiones actuales. Estudios Pedagógicos, 36(2), 333-342.

https://doi.org/10.4067/S07 18-07052010000200019

Figueroa, I. (2020). El profesionalismo dialógico como recurso para la construcción de un rol docente transformador. Paideia 64(1), en prensa.

Figueroa, I. y Muñoz, Y. (2014). La guía para la inclusión educativa como herramienta de autoevaluación institucional: Reporte de una experiencia. Revista Latinoamericana de Educación Inclusiva, 8(2), 179-198.

Figueroa, I., Soto, J. y Sciolla, B. (2016). Dinámicas de recepción, apropiación y contextualización del enfoque de la guía para la inclusión educativa en escuelas municipales de una comuna de la Región Metropolitana. Pensamiento Educativo: Revista de Investigación Educacional Latinoamericana, 53(1), 1-13. https://doi.org/10.7764/PEL.53.1.2016.4

Figueroa, I., Soto, J. y Yáñez-Urbina, C. (2019). Concepciones sobre el cambio educativo de docentes participantes de un proyecto de desarrollo inclusivo. Revista Educación, 43(1), 1-34.

García Canclini, N. (2007). De cómo la interculturalidad global debilita al relativismo. En A. Giglia, C. Garma y A. de Teresa (Comps.), ¿̇Adónde va la antropología? (pp. 39-59). Ciudad de México: UAM.

Gascón, F. y Godoy, L. (2015). Presencia e in-diferencia. Por un estatuto visual de la niñez. Revista Latinoamericana de Ciencias Sociales, Niñez y Juventud, 13(2), 645-656. https://doi.org/10.11600/1692715x.1326093014 
Gentili, P. (2011). Pedagogía de la igualdad: Ensayos contra la educación excluyente. Buenos Aires: Siglo Veintiuno.

Godoy, L. (2015). La infancia colonizada: Estatuto de (in) diferencias y visualidad. En A. Ocampo (Ed.), Lectura para todos. El aporte de la fácil lectura como vía para la equiparación de oportunidades (pp. 89-103). Santiago de Chile: Asociación Española de Comprensión Lectora y Centro de Estudios Latinoamericanos de Educación Inclusiva.

Grossberg, L. (2009). El corazón de los estudios culturales: Contextualidad, construccionismo y complejidad. Tabula Rasa, 10, 13-48. https://doi.org/10.25058/20112742.354

Guzmán, C. (2002). Reflexiones en torno a la condición estudiantil en los noventa: Los aportes de la sociología francesa. Perfiles Educativos, 24(97), 38-56.

Hall, S. (2006). Estudios culturales: Dos paradigmas. Revista Colombiana de Sociología, 27, 233-254.

Haraway, D. (1995). Ciencia, cyborgs y mujeres. La reinvención de la naturaleza. Madrid: Cátedra.

Hohti, R., Karlsson, L. y Palmu, T. (septiembre, 2013). Children as ethnographers: space for children's narrative knowing within classroom intra-action. Comunicación presentada en la Ethnography and Education Conference. Oxford.

Ibáñez-Salgado, N. y Druker-Ibáñez, S. (2018). La educación intercultural en Chile desde la perspectiva de los actores: Una co-construcción. Convergencia, 25(78), 227-249. https://doi.org/10.29101/crcs.v25i78.9788

Jorquera, C. (2015). La estructura escolar frente a los desafíos de la diversidad: Una mirada subalterna al problema de la educación inclusiva. Liminales. Escritos sobre Psicología y Sociedad, 1(8), 35-54.

Kant, I. (1994). Respuesta a la pregunta ¿Qué es la ilustración? Revista Colombiana de Psicología, 3, 7-10.

Levinson, B. (1999). Ideologías de género en una escuela secundaria mexicana: Hacia una práctica institucional de equidad. Revista Latinoamericana de Estudios Educativos, 24(2), 9-36.

Liebel, M. (2016). ¿ Niños sin niñez? Contra la conquista poscolonial de las infancias del sur global. Millcayac-Revista Digital de Ciencias Sociales, 3(5), 245-272.

Long, N. (1996). Globalización y localización, nuevos retos para la investigación rural. En S. Lara y M. Chauvet (Eds.), La inserción de la agricultura mexicana en la economía rural (pp. 37-74). Ciudad de Mexico: Plaza y Valdés.

Long, N. (2003). Development sociology: Actor perspectives. Londres: Routledge. https://doi.org/10.4324/9780203398531

Long, N. y Arce, A. (1992). Battlefields of knowledge: Interlocking of theory and practice in social research and development. Londres: Taylor \& Francis.

MacPhail, A. (2010). Listening to pupils' voices. En R. Bailey (Ed.), Physical education for learning: A guide for secondary schools (pp. 228-238). Londres: Routledge.

Martín-Barbero, J. (2002). Pistas para entre-ver medios y mediaciones. Signo y Pensamiento, 41, 1320.

Martín-Barbero, J. (2011). Razón técnica y razón política: espacios/tiempos no pensados. Revista Latinoamericana de Ciencias de la Educación, 1(1), 22-37.

Messiou, K. (2013). El compromiso con la voz del alumnado: Uso de un marco de trabajo para abordar la marginación en las escuelas. Revista de Investigación en Educación, 11(3), 97-108.

Molina, S. y Christou, M. (2009). Inclusión educativa y pedagogía crítica. Revista electrónica teoría de la educación. Educación y Cultura en la Sociedad de la Información, 10(3), 31-55. 
Muñoz, G. (2011). La democracia y la participación en la escuela: ¿Cuánto se ha avanzado desde las normativas para promover la participación escolar? Revista de Estudios y Experiencias en Educación, 10(19) 107-129.

Nieto, J. y Portela, A. (2008). La inclusión de la voz del alumno en el asesoramiento para la mejora de las prácticas educativas. profesorado. Revista de Curriculum y Formación de Profesorado, $12(1), 1-26$.

Ordóñez, C., Mejía, J. y Castellano, S. (2006). Percepciones estudiantiles sobre el fraude académico: Hallazgos y reflexiones pedagógicas. Revista de Estudios Sociales, 23, 37-44. https://doi.org/10.7440/res23.2006.03

Pérez, E. G. y Rincón, P. J. (2010). Del adulto-centrismo y otras paradojas: Una aproximación a la discriminación de la infancia y la participación infantil en la sociedad contemporánea. Papeles Salmantinos de Educación, 14, 137-156. https://doi.org/10.36576/summa.30635

Reyes, G. (2007). La práctica del psicodrama. Santiago de Chile: RIL editores.

Rogoff, B. (1997). Los tres planos de la actividad sociocultural: apropiación participativa, participación guiada y aprendizaje. En J. Wertsch, P. del Río y A. Álvarez (Eds.), La mente sociocultural. Aproximaciones teóricas y aplicadas (pp. 111-128). Madrid: Fundación Infancia y Aprendizaje.

Rojas, J. (2010). Historia de la infancia en el Chile republicano, 1810-2010. Santiago de Chile: Junji.

Rojas, S., Haya, I. y Lázaro-Vera, S. (2011). La voz del alumnado en la mejora escolar: Niños y niñas como investigadores en educación primaria. Revista de Educación, 359, 81-101.

Rojas-Bermúdez J. (1997). Teoría y técnica psicodramáticas. Barcelona: Paidós.

Rosas, K. (2012). Genealogía de los estudios culturales. Razón y Palabra, 81, 26-54.

Ruidrejo, A. (2006). De kant a Benjamin: ilustración e infancia. En W. Kohan (Comp.), Teoría y práctica en filosofía con niños y jóvenes. Experimentar el pensar, pensar la experiencia (pp. 171177). Buenos aires: Centro de Publicaciones Educativas y Material Didáctico.

Sales Ciges, A. (2017). Creando redes para una ciudadanía crítica desde la escuela intercultural inclusiva. Revista de Educación Inclusiva, 5(1), 51-67.

Salinas, C. y Stange, H. (2009). Poder y comunicación. La irresoluble tensión entre palabra, imagen y política. Perspectivas de La Comunicación, 2, 138-145.

Salinas, C. y Stange, H. (2006). ¿Viejos problemas, nuevas soluciones? Comunicación (y) política. Comunicación y Medios, 16(17), 145-157.

Santos, B. d. S (2018). Introducción a las epistemologías del sur. En M. Meneses y K. Bidaseca (Coords.), Epistemologías del sur (pp. 25-62). Buenos Aires: Clacso.

Sañudo, M. y Susinos, T. (2018). ¿Quién toma la palabra en la escuela? ¿Quién escucha? Preguntas Pertinentes desde la práctica reflexiva. Revista Latinoamericana de educación inclusiva, 12(1), 79-94. https://doi.org/10.4067/SO7 18-73782018000100079

Scolari, C. (2008). Hipermediaciones: Elementos para una teoría de la comunicación digital interactiva. Barcelona: Gedisa.

Soto, J. E., Figueroa, I. y Yáñez-Urbina, C. (2017). Asesoramiento colaborativo en escuelas municipales: Posicionamientos y desafíos del rol de amigo crítico en un proyecto de desarrollo escolar inclusivo. Revista Latinoamericana de Educación Inclusiva, 11(1), 245-264. https://doi.org/10.4067/S0718-73782017000100015

Spivak, G. (2011). ¿Puede hablar el subalterno? Buenos Aires: El cuenco de plata 
Stange, H. (2007). Hacia una reformulación de la pregunta crítica en Comunicaciones. Diálogos de la Comunicación, 75, 1-6.

Stoll, L. y D. Fink (1999). Para cambiar nuestras escuelas. Reunir la eficacia y la mejora. Barcelona: Octaedro.

Susinos, T. (2012). Presentación. Las posibilidades de la voz del alumnado para el cambio y la mejora educativa. Revista de Educación, 359, 16-23.

Susinos, T. y Ceballos, N. (2012). Voz del alumnado y presencia participativa en la vida escolar. Apuntes para una cartografía de la voz del alumnado en la mejora educativa. Revista de Educación, 359, 24-44.

Taylor, C. y Robinson, C. (2009). Student voice: Theorising power and participation. Pedagogy, Culture and Society, 17(2), 161-175. https://doi.org/10.1080/14681360902934392

Vergara, A., Peña, M., Chávez, P. y Vergara, E. (2015). Los niños como sujetos sociales: El aporte de los nuevos estudios sociales de la infancia y el análisis crítico del discurso. Psicoperspectivas, 14(1), 55-65. https://doi.org/10.5027/psicoperspectivas-Vol 14-Issue1fulltext-544

Werner, E. (2009). Cultura estudiantil y diversidad sexual: discriminación y reconocimiento de los jóvenes LGBT en la secundaria. Polisemia, 5(8), 101-110. https://doi.org/10.26620/uniminuto.polisemia.5.8.2009.101-110

Yañez-Urbina, C., Figueroa-Céspedes, I., Soto Cárcamo, J. y Sciolla Happke, B. (2018). La voz en la mirada: Fotovoz como una metodología para explorar los procesos de inclusión-exclusión desde la perspectiva del estudiantado. Pensamiento Educativo. Revista de Investigación Educacional Latinoamericana, 55(2), 1-16. https://doi.org/10.7764/PEL.55.2.2018.4

\section{Breve CV de los autores}

\section{Ignacio Figueroa-Céspedes}

Psicólogo de la Universidad de Santiago de Chile. Magister en Desarrollo Cognitivo de la Universidad Diego Portales. Doctorando en Educación de la Universidad Metropolitana de Ciencias de la Educación. Actualmente desempeña labores como académico e investigador del Centro de Desarrollo Cognitivo de la Facultad de Educación de la Universidad Diego Portales. Miembro de la Red Index for Inclusion Network. Sus líneas de investigación son inclusión educativa, desarrollo cognitivo, educación parvularia y aprendizaje profesional docente. ORCID ID: https://orcid.org/0000-0002-2756-1831. Email: ignacio.figueroa@mail.udp.cl

\section{Cristopher Yáñez-Urbina}

Psicólogo con mención en Psicología Educacional e Infanto-Juvenil de la Universidad de Santiago de Chile. Magister en Comunicación Política del Instituto de la Comunicación e Imagen de la Universidad de Chile. Doctorando en Psicología de la Pontificia Universidad Católica de Valparaíso. Actualmente desempeña labores como Asistente de Investigación y Publicaciones del Centro de Desarrollo Cognitivo de la Facultad de Educación de la Universidad Diego Portales. Sus líneas de investigación de circunscriben en los estudios críticos de infancia, educación inclusiva, y estudios de género y sexualidad. ORCID ID: https://orcid.org/0000-0001-7171-9686.Email: Cristopher.yanezu@mail.udp.cl 